NASA Technical Memorandum 103241

AIAA-90-2645

\title{
Preliminary Plume Characteristics of an Arcjet Thruster
}

David H. Manzella

Sverdrup Technology, Inc.

Lewis Research Center Group

Brook Park, Ohio

Francis M. Curran

National Aeronautics and Space Administration

Lewis Research Center

Cleveland, Ohio

Roger M. Myers

Sverdrup Technology, Inc.

Lewis Research Center Group

Brook Park, Ohio

and

Dieter M. Zube

Institut für Raumfahrtsysteme

Universität Stuttgart

Stuttgart, West Germany

Prepared for the

21 st International Electric Propulsion Conference cosponsored by the AIAA, DGLR, and JSASS

Orlando, Florida, July 18-20, 1990

\section{N/SA}

(NASA-TM-103241) PRELIMINARY PLUME CMARACTERISTICS OF AN ARCJET THRUSTER (NASA) $16 \mathrm{p}$ 
$F T=-$ 


\title{
Preliminary Plume Characteristics of an Arcjet Thruster
}

\author{
David H. Manzella \\ Sverdrup Technology, Inc. \\ Lewis Research Center Group \\ Brook Park, Ohio 44142, U.S.A. \\ Francis M. Curran \\ NASA Lewis Research Center \\ Cleveland, Ohio 44135, U.S.A. \\ Roger M. Myers \\ Sverdrup Technology, Inc. \\ Lewis Research Center Group \\ Brook Park, Ohio 44142, U.S.A. \\ Dieter M. Zube \\ Institut für Raumfahrtsysteme \\ Universität Stuttgart \\ 7000 Stuttgart 80 , W. Germany
}

An experimental investigation of a low power arcjet plume was conducted using emission spectroscopy. A laboratory-model arcjet incorporating a segmented-anode was run on simulated hydrazine at a flow rate of $5 \times 10^{-5}$ $\mathrm{kg} / \mathrm{s}$. The complete visible spectrum measured in the exit plane of the arcjet showed the presence of $\mathrm{N}_{2}, \mathrm{~N}_{2}{ }^{+}, \mathrm{NH}$, and $H$. Radial intensity profiles for the $H_{\alpha}, H_{\beta}$, and the $N H A^{3} \Pi \rightarrow X^{3} \Sigma(0,0)$ transitions at four different axial locations were measured. These line of sight intensity measurements, spaced $0.05 \mathrm{~mm}$ apart, were deconvoluted to give the radial intensity distribution using an inverse Abel transformation. The ratio between the intensities from the $H_{\alpha}$ and $H_{\beta}$ transitions indicated a non-Boltzmann energy distribution between excited states in the plume. Axial intensity profiles taken on center line indicated the decay rate of excited states in the plume. An electron number density of $2 \times 10^{13} \mathrm{~cm}^{-3}$ at the exit plane was determined based on Stark broadening of the $H_{\beta}$ line. Rotational temperatures of $750 \mathrm{~K}, 1750 \mathrm{~K}$, and $2500 \mathrm{~K}$ were determined for $\mathrm{N}_{2}, \mathrm{~N}_{2}{ }^{+}$, and $\mathrm{NH}$ respectively. The results of this investigation demonstrate that the location of the current attachment on the anode has a measurable effect on the electronically excited species in the plume and that dissociation is the dominant frozen flow loss mechanism in low power arcjets.

\section{Introduction}

Research and development on one kilowatt-class arcjets to be used for north-south station keeping of geosynchronous satellites has been underway for several years. ${ }^{1-10}$ As a result, this propulsion system has rapidly evolved from an unsteady laboratory device to a flight type unit. An extended duration lifetest has shown thruster lifetimes greater than 1000 hrs. $^{2}$ The power processing unit and other necessary components have also been developed to a flight ready status. ${ }^{3}$ Recent research efforts have been focused on addressing the remaining user concerns, answering fundamental questions about arcjet operation, and improving and extending the capabilities of the arcjet. One primary concern for arcjet system integration onto a communication satellite is the exhaust plume. Unlike the resistojet, which also heats decomposed hydrazine and expands it through a convergingdiverging nozzle, the arcjet heats the propellant to a greater extent in an arc discharge. The resulting plume is therefore more energetic than a resistojet plume. As a result of the arc, electronic excitation, ionization, and dissociation occur in the nozzle. Immediately downstream of the nozzle, the plume is a rapidly-expanding, partially-ionized plasma in which recombination and de-excitation take place. In order to quantify the effects of the plume on different spacecraft subsystems, various experimental and analytical capabilities have been employed. Experimental investigations, such as those conducted by Carney $y^{4,5}$ using electrostatic probes, have provided data on the electron number density and the electron temperature in the far field of the plume. The effect of the arcjet plume on the transmission and reception of communication signals has been modeled based on these measurements. ${ }^{6}$ Little, however, has been done to characterize the near field plume.

Many studies have been conducted to investigate arcjet operating characteristics. Experiments have demonstrated the relative performance of different geometries, ${ }^{7}$ electrical configurations, ${ }^{8}$ and operating conditions. ${ }^{9}$ These empirical studies have given 
insight into the physics governing the operation of the arcjet. However, information such as arc temperature and the nature of the arc atlachment on the anode are not well known. Such data would permit a more thorough understanding of the internal energy deposition processes which dictate the operation of the arcjet. Information about the near field plume will provide information about phenomena occurring within the arcjet and result in a more thorough understanding of the plume which is needed for an accurate impacts assessment.

This report details an experimental program initiated to characterize the near field of the arcjet plume. Measurements were taken in the near field of a laboratory-model arcjet with a segmented anode using emission spectroscopy. The effect of anode current distribution on the plume was investigated. These results, in conjunction with the results from a companion paper, ${ }^{10}$ provided information about the energy loss mechanisms in kilowatt-class arcjets.

\begin{tabular}{|c|c|}
\hline & Nomenclature \\
\hline$A$ & transition probability \\
\hline$a$ & curve fit parameter \\
\hline$b$ & curve fit parameter \\
\hline$B_{e}$ & rotational constant \\
\hline$B_{v}$ & rotational constant for vibrational state \\
\hline$c$ & velocity of light, $3.0 \times 10^{8} \mathrm{~m} / \mathrm{s}$ \\
\hline$C_{e m}$ & constant, (eqn 7) \\
\hline$E_{H}$ & ionization energy for hydrogen, \\
\hline$J$ & variable in transformed domain \\
\hline$G$ & Fourier transform of I \\
\hline$\hat{G}$ & discretized values of $G$ \\
\hline$g$ & statistical weight \\
\hline$h$ & Planck's constant, $6.626 \times 10^{-34} \mathrm{~J} \mathrm{~s}$ \\
\hline$I$ & intensity, $\mathrm{W}$ \\
\hline$\hat{I}$ & discretized values of I \\
\hline$i$ & index for summation \\
\hline$J$ & rotational quantum number \\
\hline 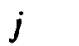 & $\sqrt{-1}$ \\
\hline$J_{0}$ & zero order Bessel function \\
\hline$k$ & Boltzmann's constant, $1.380 \times 10^{-23} \mathrm{~J} / \mathrm{K}$ \\
\hline$l$ & index for summation \\
\hline$m$ & species mass, $\mathbf{g}$ \\
\hline$N$ & number of discrete points \\
\hline$N_{e}$ & electron number density, $\mathrm{cm}^{-3}$ \\
\hline$n$ & quantum number \\
\hline$Q_{r}$ & partition function, (eqn 8) \\
\hline$r$ & radial distance, $\mathrm{cm}$ \\
\hline$T$ & temperature \\
\hline$T_{\alpha x}$ & excitation temperature, $\mathrm{K}$ \\
\hline$T_{e}$ & electron temperature, $\mathbf{K}$ \\
\hline$T_{\text {rot }}$ & rotational temperature, $\mathbf{K}$ \\
\hline $\mathbf{v}$ & vibrational quantum number \\
\hline$z$ & species charge +1 \\
\hline$\alpha_{\varepsilon}$ & rolational constant \\
\hline
\end{tabular}

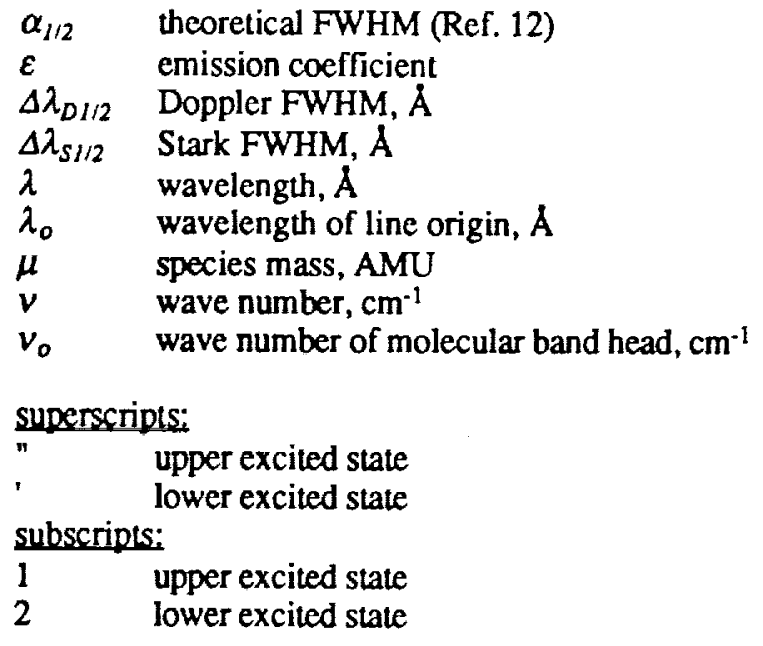

Analysis

Excilation temperature. Within a plasma, energetic collisions can result in electronic excitation. The population of an electronically excited state is proportional to the population of Jower energy states, the electron number density, the electron energy distribution, and the appropriate collisional cross sections. As the electronically excited species relax, quantized energy is spontaneously emitted. The emitted energy from this radiative de-excitation is proportional to the population of the excited state and the probability that the electronic transition will occur. In an optically thin plasma, the emitted energy passes through the plasma without being reabsorbed. When the energy distribution between electronically excited states can be described by a Boltzmann equilibrium distribution, and the plasma is optically thin, the ratio between the energy emitted from two different transitions can be written as:

$$
\frac{I_{1}}{I_{2}}=\frac{A_{1} g_{1} \lambda_{2}^{3}}{A_{2} g_{2} \lambda_{1}^{3}} \exp \left(-\left(E_{\Gamma} E_{2}\right) / k T_{\infty}\right)
$$

From this expression the excitation temperature, $T_{e x}$, can be calculated. The significance of this temperature is dependent on the degree of equilibrium within the plasma. For an optically thin plasma, if collisional processes occur much more rapidly than radiative processes, the plasma is considered to be in local thermodynamic equilibrium (LTE). For a plasma in LTE, any point can be described by the local values for temperature, density, and chemical composition. However, in many cases the conditions for LTE are not met. In a non-LTE plasma all equilibrating collisional processes are not significantly faster than other plasma processes. As a result, the energy distribution for different species can not be described by a single temperature and multiple temperatures are employed. Atoms may have an excitation temperature and a kinetic temperature. Molecules may have several different temperatures including the rotational temperature, vibrational 
temperature, excitation temperature, and kinetic temperature. In this study, the $H_{\alpha}(6562.8 \AA)$ and $\mathrm{H}_{\beta}(4861.3 \AA)$ electronic transitions from the Balmer series were used to calculate an excitation temperature based on the line ratio method presented above.

The suitability of the assumption of a Boltzmann energy distribution between the third and fourth energy levels of hydrogen, ie. the upper excited states of $\mathrm{H}_{\alpha}$ and $\mathrm{H}_{\beta}$, can be estimated using the criteria for partial local thermodynamic equilibrium (PLTE) presented by Griem, see Ref. 11. For an optically thin plasma, this requires the electronic collisional rates for the upper excited states to be at least ten times faster than the radiative decay rates. The required electron number density for equilibrium between electrons and upper excited states in a hydrogenic plasma depends on the electron temperature.

$$
\mathrm{N}_{\mathrm{e}} \geq 7 \times 10^{18} \frac{\mathrm{z}^{7}}{\mathrm{n}^{17 / 2}}\left(\frac{\mathrm{kT}_{\mathrm{e}}}{\mathrm{z}^{2} \mathrm{E}_{\mathrm{H}}}\right)^{1 / 2}
$$

Electron number density. Spectral line emissions are broadened by several different mechanisms. These include Doppler broadening and Stark broadening. Instrumental limitations can also lead to apparent broadening. Doppler broadening is the result of a frequency shift of the emitted light due to the motion of the radiating particle in relation to the observer. Because the radiating species have some relative statistical velocity distribution, not all the emitted light is shifted the same amount and the spectral line is broadened. This relative velocity distribution describes the kinetic temperature of the emitting species. For a Maxwellian velocity distribution, the line shape due to Doppler broadening will be Gaussian and the full width at half maximum (FWHM) for the line can be written as:

$$
\Delta \lambda_{\mathrm{D} 1 / 2}=\left[(8 \mathrm{kT} \ln 2) /\left(\mathrm{mc}^{2}\right)\right]^{1 / 2} \lambda_{0}
$$

Doppler line widths for several different temperatures are shown in Table $\mathrm{I}$ for the $\mathrm{H}_{\beta}$ line.

Table I: Calculated FWHM due to Doppler

\begin{tabular}{cc}
\hline \multicolumn{3}{c}{ Broadening } \\
\hline $\mathrm{T}, \mathrm{K}$ & $\Delta \lambda_{\mathrm{D} 1 / 2}, \AA$ \\
\hline 1000 & 0.078 \\
2000 & 0.110 \\
3000 & 0.135 \\
4000 & 0.156 \\
5000 & 0.174 \\
6000 & 0.191 \\
7000 & 0.206 \\
8000 & 0.220 \\
9000 & 0.233 \\
10000 & 0.246 \\
\hline
\end{tabular}

Stark broadening produces Lorentzian line shapes and is caused by the interactions of the emitters with charged particles. Theories have been developed to predict how ions or electrons affect a line profile. ${ }^{12}$ The functional dependency of the electron number density on the FWHM can be simplified: ${ }^{12}$

$$
\Delta \lambda_{\mathrm{S} / 2}=2.50 \times 10^{\circ} \alpha_{1 / 2} \mathrm{~N}_{\mathrm{e}}^{2 / 3}
$$

This expression was used to predict the FWHM for the $\mathrm{H}_{\beta}$ line as a function of electron number density. Calculated values are displayed in Table II.

Table II: Calculated FWHM due to Stark Broadening

\begin{tabular}{ll}
\hline $\mathrm{N}_{\mathrm{e}}, \mathrm{cm}^{-1}$ & $\Delta \lambda_{\mathrm{S} 1 / 2}, \AA$ \\
\hline $1 \times 10^{12}$ & $5.56 \times 10^{-3}$ \\
$5 \times 10^{12}$ & $4.58 \times 10^{-2}$ \\
$1 \times 10^{13}$ & $1.14 \times 10^{-1}$ \\
$5 \times 10^{13}$ & $9.38 \times 10^{-1}$ \\
$1 \times 10^{14}$ & $2.33 \times 10^{0}$ \\
\hline
\end{tabular}

The combined effects of Doppler and Stark broadening can be described using a Voigt profile. ${ }^{12}$ If the kinetic temperature is known, the contribution to the Voigt profile due to Doppler broadening can be estimated. From the ratio between the observed line width and the estimated Doppler line width, the amount the line was Stark broadened can be determined. This gives the electron number density.

Rolational Temperature. The different vibrational and rotational molecular energy states give rise to vibrational-rotational band spectra. During the transition from a higher electronic energy level to a lower electronic energy level, the vibrational energy state may change. Additionally, within any vibrational state there are many discrete rotational states. These different rotational states, separated by small energy differences, result in the many individual lines characteristic of the molecular spectra. From a rotating vibrator approximation, the wave numbers for the different rotational lines for the three different branches can be estimated via the following equations: ${ }^{13}$

$$
\begin{aligned}
v= & v_{0}+2 B^{\prime}{ }_{v}+\left(3 B^{\prime}{ }_{v}-B^{\prime \prime}{ }_{v}\right) J "+\left(B^{\prime}{ }_{v}-B^{\prime \prime}{ }_{v}\right) J^{\prime \prime} \\
v & =v_{0}+\left(B^{\prime}{ }_{v}-B^{\prime \prime}{ }_{v}\right) J^{\prime \prime}+\left(B^{\prime}{ }_{v}-B^{\prime \prime}{ }_{v}\right) J^{\prime \prime} \\
v & =v_{0}-\left(B^{\prime}{ }_{v}+B^{\prime \prime}{ }_{v}\right) J^{\prime \prime}+\left(B^{\prime}{ }_{v}-B^{\prime \prime}{ }_{v}\right) J^{\prime \prime}
\end{aligned}
$$

In these, $B_{v}$ is a vibrational constant that varies with the vibrational quantum number.

$$
B_{v}=B_{e}-\alpha_{e}\left(v+\frac{1}{2}\right)
$$

Assuming a constant transition probability for all the lines of a rotational-vibrational band and a Boltzmann energy distribution betwcen the different rotational levels within a given vibrational state, the 
intensity of each of the rotational lines can be calculated,

$$
I=\frac{C_{o v} v^{4}}{Q_{r}}\left(J^{\prime}+J^{\prime \prime}+1\right) \exp \left[B^{\prime} v^{\prime}\left(J^{\prime}+1\right) h c / k T\right]
$$

where $\mathrm{C}_{\mathrm{em}}$ is a constant and the partition function $\mathrm{Q}_{\mathrm{r}}$ can be approximated by

$$
\mathrm{Q}_{\mathrm{r}}=\frac{\mathrm{kT}}{\mathrm{hcB}}
$$

Using equations (5) - (8) the complete molecular spectra for a particular transition can be calculated. The temperature used to determine the intensity defines the distribution of rotational energies in the vibrational state and will be referred to as the rotational temperature. The fact that the rotational temperature determines the intensity distribution in a rotational-vibrational band was used to determine the rotational temperature from the measured spectra. For this an iterative process was used to determine the temperature giving the best agreement between the calculated band and the measured band.

\section{Apparatus}

An experimental system was designed and built to take spectroscopic measurements in the plume of kilowatt-class arcjets. The arcjet was installed within a cylindrical vacuum chamber measuring $1.5 \mathrm{~m}$ in diameter by 5 meters in length. The chamber's pumping system consisted of four $0.82 \mathrm{~m}$ oil diffusion pumps (each rated at $30,000 \mathrm{l} / \mathrm{s}$ ), a lobe type blower, and two piston type roughing pumps. The ambient pressure during arcjet operation was nominally $0.067 \mathrm{~Pa}$.

The arcjet fired along the major axis of the tank and was mounted on a two-axis, computer-controlled, linear actuator system. This actuator system was capable of moving the thruster vertically and horizontally relative to the collection optics with a positional accuracy of about $0.025 \mathrm{~mm}$. Both actuators were mounted on a single platform vibrationally isolated from the vacuum chamber.

The optical system, shown in Figure 1, was designed to image the side view of the plume on the entrance slit of a monochrometer. The overall magnification of the system was 2.8 . The primary collector was a $150 \mathrm{~mm}$ diameter parabolic mirtor with a $406 \mathrm{~mm}$ focal length. This mirror was positioned $1.4 \mathrm{~m}$ from the center line of the plume. resulting in a solid angle of $8.9 \times 10^{-3}$ steradians. A second parabolic minor with a diameter of $100 \mathrm{~mm}$ and a focal length of $228 \mathrm{~mm}$ was used to provide the desired magnification and focus the image of the plume on the entrance slit of a $0.64 \mathrm{~m}$ Czemy-Tumer type monochrometer. Data were taken with the entrance slit of the spectrometer opened to a height of $3 \mathrm{~mm}$ and a width of 10 microns. Consequently, the cross sectional dimensions of the volume of the plume measured at each point was approximately 1 $\mathrm{mm}$ in height by 3.6 microns in width. The spectrometer had a $110 \mathrm{~mm} \times 110 \mathrm{~mm}$ holographic ruled grating with 2400 grooves $/ \mathrm{mm}$. This grating was blazed for use between $2500 \AA$ and $7500 \AA$. The theoretical linear dispersion of this spectrometer system was $0.06 \mathrm{~A} / \mathrm{mm}$.

Two different photodetectors were used. A side-on photo-multiplier tube (PMT) with a quartz window and a multi-alkali photocathode was mounted on the axial exit port of the spectrometer, and a 1025 element intensified linear photodiode array was mounted on the lateral exit port. Both detectors were thermoelectrically cooled to minimize the dark current signal.

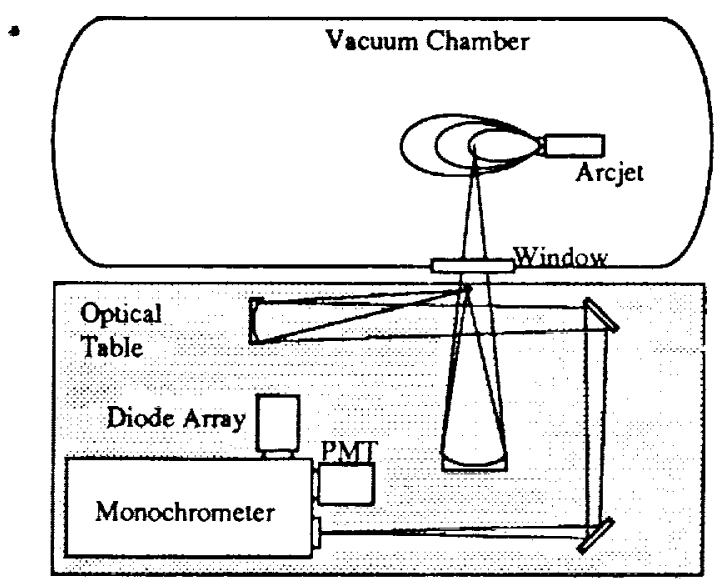

Figure 1. Experimental Apparatus and Optical Configuration, Not to Scale

The arcjet used in this investigation was a laboratory-model thruster with a segmented anode/nozzle. The thruster, which has been used in other investigations, has been described in detail elsewhere. ${ }^{10}$ Briefly, the major components include a $2 \%$ thoriated tungsten cathode $3.2 \mathrm{~mm}$ in diameter terminated in a cone with a 30 degree half angle (which corresponds to the convergence half angle of the anode). The propellant was injected tangentially through an annular injection disk to aid in arc stabilization.

A radiation-cooled, segmented anode/nozzle consisting of alternating conducting and nonconducting segments was used. A simplified cross-sectional diagram of both electrodes is shown in Figure 2. The size and contour of the anode were chosen to approximate a standard laboratory-model configuration. The nozzle diverged with a 20 degree half angle, had constrictor dimensions of $0.64 \mathrm{~mm}$ in diameter by $0.25 \mathrm{~mm}$ in length, and an area ratio of 225:1. The constrictor segments were numbered one through five. Segment number one corresponded to the furthermost upstream segment which included the 
constrictor. Segment number five corresponded to the last downstream segment. Segments one and five were fabricated from $2 \%$ thoriated tungsten. Segments two, three, and four were fabricated from tantalum. The nonconducting insulators between the segments were fabricated from boron nitride. The entire anode was held in compression with four bolts, each with a spring to accommodate the thermal expansion of the anode.

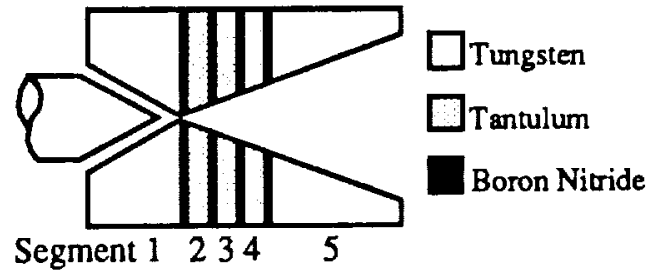

Figure 2: Simplified Schematic of Electrode Configuration

The electrical configuration of the anode was such that any of the segments could be manually switched in or out of the circuit. The current through each segment was measured with a Hall effect current probe. The voltage potential between the cathode and each segment was measured using a digital multimeter. The arcjet was run with a pulse-widthmodulated, laboratory-model power supply. Arc breakdown was initiated by a $3 \mathrm{kV}$, microsecond duration starting pulse.

All tests were conducted using a propellant mixture of hydrogen and nitrogen in a 2:1 ratio. This propellant mixture simulated the decomposition products of hydrazine. The flow rate of each gas was regulated with a mass flow controller.

\section{Procedure}

For all tests the arcjet was run with a total propellant flow rate of $5.0 \times 10^{-5} \mathrm{~kg} / \mathrm{s}$ at a total current of 10 Amperes. These are typical conditions anticipated for flight application. Prior to each series of experiments the flow meters and the current probes were calibrated. In addition, the power supply was operated with a resistive load to verify the 10 Ampere output. Three different electrical configurations were chosen to minimize the total number of experimental runs due to the limited durability of the individual anode segments. The electrical configuration for each of these three configurations is shown in Table III.

Table III: Electrical Configurations

\begin{tabular}{lccccc}
\hline \hline Segment & 1 & 2 & 3 & 4 & 5 \\
\hline Configuration 1 & $\mathrm{O}$ & $\mathrm{X}$ & $\mathrm{X}$ & $\mathrm{X}$ & $\mathrm{X}$ \\
Configuration 2 & $\mathrm{O}$ & $\mathrm{O}$ & $\mathrm{O}$ & $\mathrm{O}$ & $\mathrm{X}$ \\
Configuration 3 & $\mathrm{X}$ & $\mathrm{X}$ & $\mathrm{X}$ & $\mathrm{X}$ & $\mathrm{O}$ \\
$\mathrm{X}$ denotes conducting, O denotes nonconducting
\end{tabular}

Before pump down of the vacuum chamber an absolute intensity calibration was performed using the linear diode array. This permitted calculation of accurate intensity ratios for the determination of the excitation temperatures from $\mathrm{H}_{\alpha}$ and $\mathrm{H}_{\beta}$. A tungsten ribbon lamp was used as the calibration light source. The lamp was placed inside the chamber at the same location as the arcjet. The temperature of the filament was measured with a two color pyrometer. Simultaneously, the intensity of the incident radiation was measured at the wavelengths of interest using the diode array. The energy flux through the entrance slit of the spectrometer at a particular wavelength was then calculated using Planck's spectral distribution of emissive power for gray bodies. The emissivity used in this calculation was from a temperature and wavelength dependent polynomial expression from Larrabee. ${ }^{14}$ The comparison of the measured values with the calculated energy flux resulted in a calibration of the measured intensity versus actual intensity.

After the intensity calibration was completed, the thruster was installed on the actuator system and realigned relative to the optics. A small incandescent light bulb was placed inside the anode such that a portion of the filament was visible from the side view. The thruster was then positioned horizontally and vertically relative to the primary collector until the image of the light bulb filament was properly aligned on the entrance slit. At this time the second parabolic mirror was adjusted such that the image was properly focused. The light was then removed and the chamber was pumped down to the high vacuum condition.

Prior to taking spectroscopic data the arcjet was run until steady-state conditions were obtained. This was determined based on constant voltage/current characteristics, constant anode/nozzle temperature as measured with a two-color pyrometer, and negligible additional movement of the nozzle exit plane due to thermal expansion. Steady-state anode temperatures of approximately $1460 \mathrm{~K}$ were measured on the outer surface. Thermal expansion of the arcjet was monitored optically using the spectroscopic system. The arcjet was positioned such that the nozzle exit plane coincided with the entrance slit of the spectrometer. However, as the temperature of the arcjet approached steady state, the arcjet's length increased due to thermal expansion and the nozzle exit plane was no longer imaged on the entrance slit. Due to limited conductive paths between the segmented anode and the anode housing it took approximately 30 minutes for this equilibrium condition to be satisfied. The thruster was then repositioned axially until the nozzle exit plane was again properly imaged on the spectrometer's entrance slit.

Intensity measurements were then taken with either the diode array or the photomultiplier tube. The 
intensity measurements taken with the diode array consisted of radial and axial scans through the plume for a given spectral region. Radial scans were taken at four different axial locations: the exit plane, and $2.5,5.0$, and $7.5 \mathrm{~mm}$ downstream of the exit plane. Each radial scan consisted of 257 spectra separated by $0.05 \mathrm{~mm}$. The axial scans were taken along the plume centerline. Each axial scan consisted of 200 spectra, each separated by $0.05 \mathrm{~mm}$. For each of the axial and radial scans the spectral regions containing the $\mathrm{H}_{\alpha}$ and $\mathrm{H}_{\beta}$ emissions were monitored. Radial and axial scans were also taken for $\mathrm{NH} \mathrm{A}^{3} \Pi \rightarrow X^{3} \Sigma(0,0)$ and $(1,1)$ transitions. One radial scan was taken at the nozzle exit plane for $\mathrm{N}_{2}{ }^{+} \mathrm{B}^{2} \Sigma_{\mathrm{u}} \rightarrow \mathrm{X}^{2} \Sigma_{8}(0,0)$ and $(1,1)$ transitions. In most cases total intensities were measured by integrating over an interval two hundred times the full width at half maximum of any particular line as discussed in Ref 12. The dark current signal from the detector was subtracted from the measured total intensity as was the signal due to continuum radiation. The uncertainty in the intensities measured with the linear diode array were found to be within \pm 5 percent based on observed temporal fluctuations. The data taken with the photomultiplier tube consisted of wavelength scans at one axial location on the plume centerline. These data covered the region from $3200 \AA$ to $7200 \AA$. Data were taken at $0.25 \AA$ increments and the intensity signal was integrated over 0.5 seconds.

As previously indicated, both the axial and radial scan data were taken using the linear diode array. A computer controlled data acquisition and control scheme was used to take these data. Figure 3 is a flow chan showing the sequence of events for a scan. Two computers were used. The first computer controlled the movements of the arcjet and triggered the second computer to acquire data once the number of data points, the physical spacing between points, and the duration of each exposure were entered. The first computer triggered data acquisition via a TTL pulse. This pulse was sent to a pulse generator which triggered the diode array through the diode array controller which was interfaced with the second computer. The data were then saved on this computer before the first computer moved the thruster to the next location and again triggered the diode array. This process continued until the full scan was completed. After a particular scan was completed the electrical configuration was changed and the process was repeated.

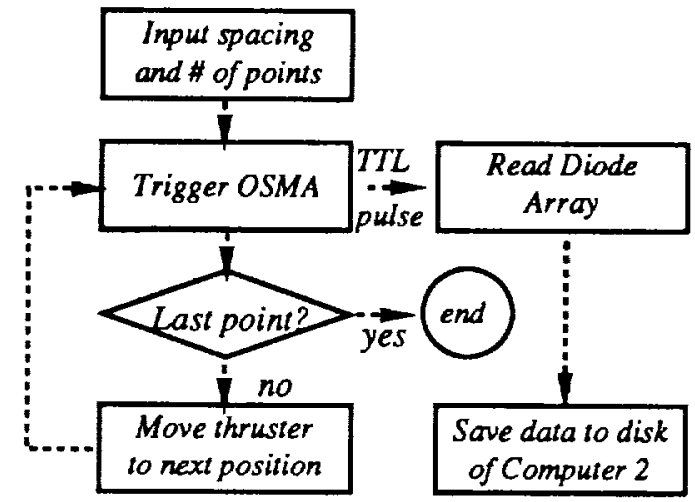

Figure 3: Data Acquisition System Flow Chart

\section{Results and Discussion}

Specira. The entire visible spectrum, for configuration 1, measured looking through the plume at the nozzle exit plane on centerline, is shown in Figure 4. The most prominent emissions were identified based on tabulated data. ${ }^{15,16}$ The resolution of the spectroscopic system was sufficient to resolve the molecular band structure. The $\mathrm{H}_{\alpha}$ and $\mathrm{H}_{\beta}$ lines are not shown to scale in order to allow sufficient detail of the lower intensity emissions. The presence of atomic hydrogen, molecular nitrogen ions, and $\mathrm{NH}$ at the exit plane indicates that dissociation, ionization, and recombination occur in the anode/nozzle. There was no significant difference between this spectrum and spectra measured in the plume of a standard laboratory model arcjet with a solid tungsten anode operating under similar conditions. This indicates that the physical processes occurring within the plume in both cases were very similar and that the segmented anode arcjet accurately simulates a standard arcjet. However, differences may exist between this spectrum and the one obtained from an arcjet run on the actual decomposition products of hydrazine. The

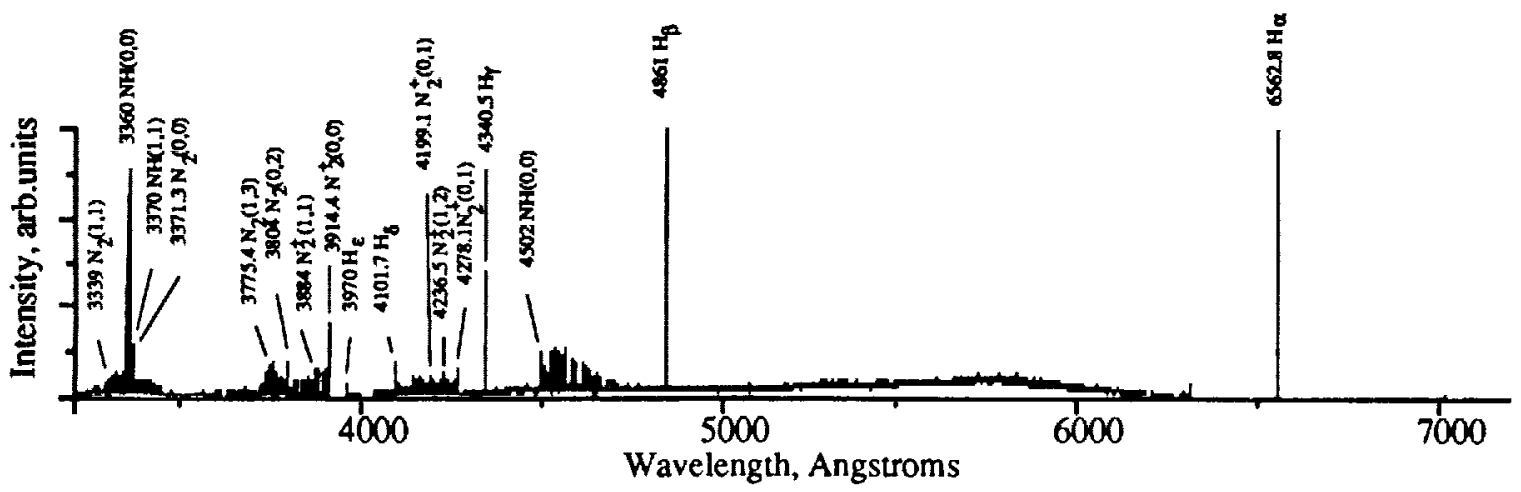

Figure 4: Complete Spectrum measured at the Exit Plane, Configuration 1 
continuum radiation between 5000 and $6500 \AA$ was partially the result of the dark current signal from the photomultiplier tube and ambient light

Radial Intensity Profiles. The radial intensity variation from the $\mathrm{H}_{\beta}$ emission line at $4861 \AA$ for four different axial positions is shown in Figure 5. These data represent total intensities integrated over the entire line. At any given spatial location the cross sectional dimensions of the portion of the plume imaged on the entrance slit of the spectrometer was 1 micron wide by $1 \mathrm{~mm}$ high. Because the arcjet was moved $0.05 \mathrm{~mm}$ vertically between data points, these total intensities represent moving averages. The curves fit to each of the radial profiles were calculated assuming a radial intensity distribution of the form $I=I_{\max } a^{-b x^{2}}$.

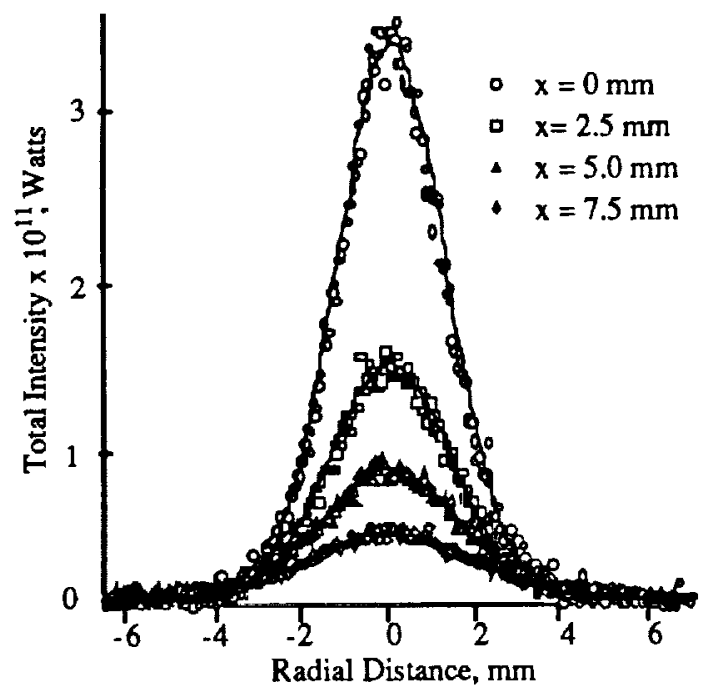

Figure 5: Radial Intensity Profiles of $\mathrm{H}_{\beta}$ emission at the Nozzle Exit Plane

The radial intensity profiles wcre inverted using the inverse Abel transformation discussed in the Appendix. Figure 6 is a sample of an inverted profile for the $\mathrm{H}_{\alpha}$ emission line. There is a significant amount of scatter in the inverted values near centerline. Emissions from the central portion of the plume constituted only a small fraction of the total light observed along a line of sight. As a result, small fluctuations in the measured total intensity near centerline propagated through the inversion, resulting in larger fluctuations in the inverted intensities near centerline. In every case, the curve fit to the radial intensity profile was also inverted. Each point of the inverted radial intensity profile is proportional to the population density of that particular electronic state. The radial decrease in inverted intensity shown in Figure 6 shows how the population of this particular electronic state varies with radial distance. The populations were essentially undectactable at radii greater than about $4 \mathrm{~mm}$.

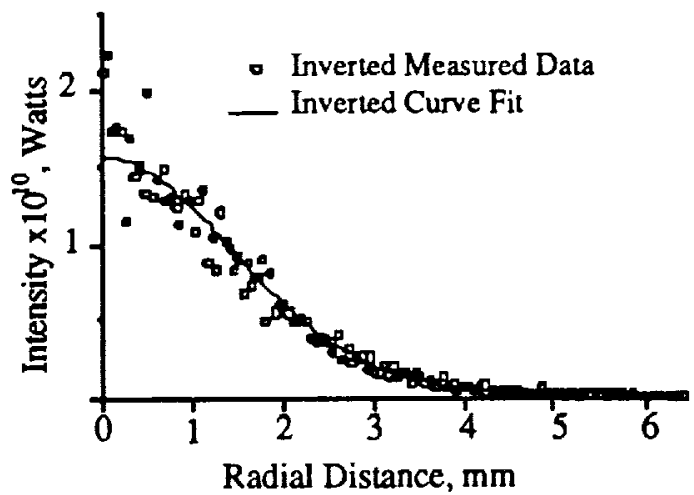

Figure 6: Inverted Intensity from $\mathrm{H}_{\alpha}$, Configuration $2,2.5 \mathrm{~mm}$ from the exit plane

Axial Intensity Profiles. The axial variation in the intensity was measured along nozzle centerline for $H_{\alpha}, H_{\beta}$, and $\mathrm{NH}$ for each of the three electrical configurations. Figure 7 shows the axial variation of $\mathrm{H}_{\alpha}, \mathrm{H}_{\beta}$ and $\mathrm{NH} \mathrm{A} \Pi \rightarrow \mathrm{X}^{3} \Sigma(0,0)(3361 \AA)$ for configuration 1 . The plot has a semi-log scale and the observed linearity indicates the decays were exponential. The intensities for $\mathrm{H}_{\alpha}$ and $\mathrm{H}_{\beta}$ are total intensities. The intensities for $\mathrm{NH}(0,0)$ are peak intensities and the units are arbitrary. This is because themolecular spectra from the $\mathrm{NH} \mathrm{A}^{3} \Pi \rightarrow \mathrm{X}^{3} \Sigma(1,1)$ transition at $3370 \AA$ and the bands from $\mathrm{N}_{2} \mathrm{C}^{3} \Pi \rightarrow$ $\mathrm{B}^{3} \Pi(0,0)$ and $(1,1)$ transition at $3371.3 \AA$ and 3339 $A$ overlapped the $\mathrm{NH}(0,0)$ rotational-vibrational band, greatly complicating a determination of the total energy of any of these bands.

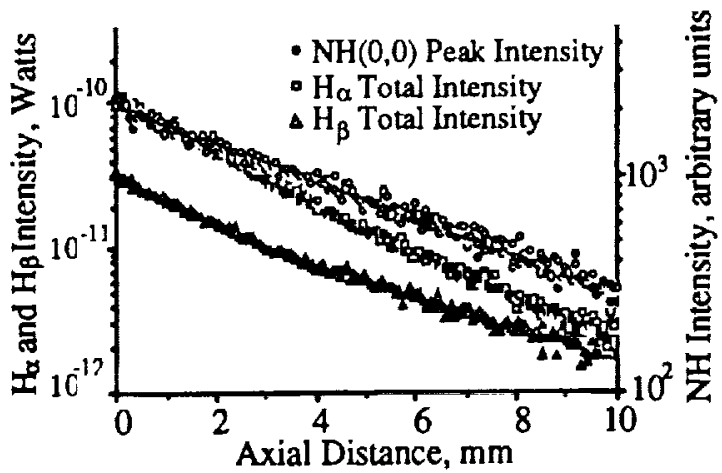

Figure 7: Axial Intensity Variation of $\mathrm{H}_{\alpha}, \mathrm{H}_{\beta}$ and $\mathrm{NH}(0,0)$ for Configuration 1

For a particular electronic transition, the axial decay rate of the emitted light intensity can be estimated based on the rate of expansion and the relative rates at which the upper excited state was populated and depopulated. The populating mechanisms are electronic excitation from lower energy states and radiative decay from higher energy states. An estimation of the rate with which the state is populated due to collisional excitation required an estimate of the number densities of the collisional 
partners and appropriate collisional cross sections. An estimation of the rate with which the state is populated due to radiative decay from higher energy states requires an estimate of the number densities for each of these and the appropriate transition probabilities. Depopulation occurs because of radiative decay, collisional de-excitation, and collisional excitation to higber energy states.

Based on the radiative lifetimes for the transitions measured, the times needed for the observed intensity decreases were calculated assuming that the decrease in intensity was solely the result of radiative decay, i.e. no collisional de-excitation and no significant repopulation in the plume. The calculated times for the observed rate of decay for $\mathrm{NH}$ indicates a velocity approximately three times lower than for atomic hydrogen, if the NH molecules were expanded at the same rate or a slower rate than the atomic hydrogen. Because velocity is inversely proportional to the square of particle mass, higher velocities were expected for the lighter species. However, the times calculated for atomic hydrogen using the radiative lifetimes of $\mathrm{H}_{\alpha}$ and $\mathrm{H}_{\beta}$ were significantly different.

If the decay would soley be governed by the transition probability, the shorter time calculated for the axial decay of $\mathrm{H}_{\alpha}$ would indicate that its upper energy state, the third energy level, was repopulated at a higher rate than the upper energy state of $\mathrm{H}_{\beta}$, the fourth energy level. The rate of collisional excitation for these two states was estimated from the excitation cross sections as presented by Drawin. ${ }^{17}$ The estimated rate of repopulation due to collisional excitation from the ground state indicated that, while collisional repopulation of the third energy level occurred more frequently than repopulation of the fourth energy level, significant collisional repopulation of these states was unlikely. Therefore, repopulation due to radiative decay of higher energy states was the significant repopulating mechanism.

Based on a consideration of the transition probabilities and oscillator strengths for various other hydrogen transitions ${ }^{15}$ it was likely that third and fourth energy levels were populated at comparable rates from decay of the fifth energy level and above. However, the preferential repopulation of the third energy level could occur as a result of the $P_{\alpha}$ transition, from energy level 4 to 3 . This transition, which is not visible $(18751 \AA)$, accounted for the observed rate of decay for $H_{\alpha}$. Since the transition probability for $P_{\alpha}$ was smaller than that for $H_{\alpha}$, the dominant rate controlling step in the decay of energy level 3 was the rate at which it was repopulated from the fourth energy level. Calculations of the time assosciated with the intensity decrease of $H_{\alpha}$ using the decay rate of $P_{\alpha}$ agreed with the values calculated for
$H_{\beta}$, because the radiative lifetime for $P_{\alpha}$ is close to that of $\mathrm{H}_{\beta}$.

Electron Number Density and Electronic Excitation Temperature. The determination of the radial distribution of the electron number density from the full width at half maximum of the $\mathrm{H}_{\beta}$ line due to Stark broadening was not possible using the data taken with the linear diode array due to instrumental broadening. For all cases the measured FWHM was $0.4 \AA$ indicating narrower lines could not be resolved. Calculations verified that this was the result of the resolution of the linear diode array. Although the limited resolution of the system precluded a determination of local electron number densities, a FWHM of $0.4 \AA$ permitted a calculation of an absolute upper limit for the electron number density. As discussed previously, to obtain the electron number density from a line profile, the line must be deconvoluted to determine the amount of Doppler and Stark broadening. To determine the amount the line was broadened due to Doppler broadening, a kinetic temperature for electronically excited hydrogen of $5000 \mathrm{~K}$, based on measurements to be discussed in the following section, was used. Using this temperature, the calculated electron number derisity for an $\mathrm{H}_{\beta}$ line whose FWHM is $0.4 \AA$ was $2.2 \times 10^{13}$ $\mathrm{cm}^{-3}$. Thus the electron number densities at each point measured were below this value.

Subsequent to this test, the FWHM for the $\mathrm{H}_{\beta}$ line was measured in the plume of an identical laboratory-model thruster with a solid anode operating at the same conditions. These later measurements were taken using the photomultiplier tube. As a result of the higher resolution of this detector, the instrumental broadening was reduced and the $\mathrm{H}_{\beta}$ line was resolved. The FWHM measured at the exit plane on centerline was $0.34 \mathrm{~A}$. These measurements were non-inverted, line-of-sight intensities values. For a temperature of $5000 \mathrm{~K}$, this FWHM corresponds to an electron number density of $1.8 \times 10^{13} \mathrm{~cm}^{-3}$.

As noted in the Analysis section, if an equilibrium or Boltzmann distribution exists between the different electronically excited states of an atom, the line intensity method can be used to determine the electronic excitation temperature. Temperature profiles were calculated using the inverted intensities from $H_{\alpha}$ and $H_{\beta}$ emission lines assuming equilibrium. For electrical configurations 1 and 2 flat temperature profiles were obtained with temperatures of approximately $5000 \mathrm{~K}$ and $5500 \mathrm{~K}$. While these temperatures seem reasonable, the intensities measured for electrical configuration 3 produced negative values for electronic excitation temperature. 
Based on calculations of the rate of collisional deexcitation from the excitation cross sections, ${ }^{17}$ radiative decay was the dominate de-excitation process. Because the radiative lifetime of $H_{\alpha}$ is approximately five times less than that for $\mathrm{H}_{\beta}$, one would expect the upper excited state of $\mathrm{H}_{\alpha}$ should be depopulated more rapidly than $\mathrm{H}_{\beta}$. For this case, the line ratio method would yield falsely high temperatures for a slight deviation from equilibrium and negative values for more substantial deviations. The ratio of the intensities from the two transitions is shown in Figure 8. The increasing intensity ratio with distance indicated a more rapid depopulation of $\mathrm{H}_{\mathbf{\alpha}}$.

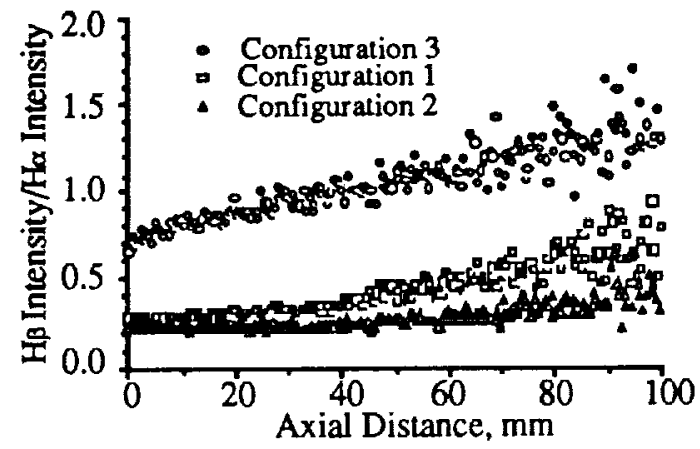

Figure 8: Axial $\mathrm{H}_{\beta} / \mathrm{H}_{\alpha}$ Intensity Ratio

For an assumed electron temperature of $5600 \mathrm{~K}$ $(0.5 \mathrm{eV})$, electron number densities greater than $10^{14}$ $\mathrm{cm}^{-3}$ are required to satisfy the conditions for PLTE presented in the analysis section. This is approximately an order of magnitude higher than the electron number density estimated from the observed $\mathrm{H}_{\beta}$ line width. The negative temperature values calculated from the $\mathrm{H}_{\alpha}$ and $\mathrm{H}_{\beta}$ line intensities for configuration 3 and the measured electron number density indicate a non-Boltzmann energy distribution between these upper excited states. As a result, the line intensity ratio is not valid for the determination of excitation temperatures for any of the configurations.

Rotational Temperature Molecular spectra from $\mathrm{N}_{2}, \mathrm{~N}_{2}{ }^{+}$, and $\mathrm{NH}$ were used to determine rotational temperatures. The thruster was operated in configuration 1 for all of these data. The rotational temperatures determined from the listed electronic transitions and the location of the band heads are listed in Table IV. The intensities of these molecular spectra were measured with the photomultiplier tube. These values were not inverted due to the added complexity of inverting an entire molecular band prior to the temperature analysis. Therefore, the intensities are line of sight averages across the nozzle exit plane on centerline. As a result, the temperatures are mean values for the plasma in this region. Based on the radial intensity profiles, the signal was averaged over approximately $5 \mathrm{~mm}$. The uncertainty was estimated to be $\pm 50 \mathrm{~K}$. No conclusive evaluation of the rotational temperature from the NH $A^{3} \Pi \rightarrow X^{3} \Sigma(0,0)$ and $(1,1)$ transitions was possible because of the overlap of these two bands and the two $\mathrm{N}_{2}$ bands in this region mentioned previously. However, a temperature near $2500 \mathrm{~K}$, measured for NH from the $\mathrm{C}^{1} \Pi \rightarrow \mathrm{B}^{1} \Sigma$, transition was likely.

Table IV. Electronic Transitions and Rotational Temperatures

\begin{tabular}{cccc}
\hline \multicolumn{2}{c}{ Electronic Transition } & $\lambda, \AA$ & $\mathrm{T}_{\text {rol }}, \mathrm{K}$ \\
\hline $\mathrm{N}_{2}$ & $\mathrm{C}^{3} \Pi_{\mathrm{u}} \rightarrow \mathrm{B}^{3} \Pi_{\mathrm{g}}(0,2)$ & 3804.9 & 800 \\
$\mathrm{~N}_{2}$ & $\mathrm{C}^{3} \Pi_{\mathrm{u}} \rightarrow \mathrm{B}^{3} \Pi_{\mathrm{g}}(1,3)$ & 3755.4 & 700 \\
$\mathrm{~N}_{2}{ }^{+}$ & $\mathrm{B}^{2} \Sigma_{\mathrm{u}} \rightarrow \mathrm{X}^{2} \Sigma_{\mathrm{g}}(0,0)$ & 3914.4 & 1800 \\
$\mathrm{~N}_{2}{ }^{+}$ & $\mathrm{B}^{2} \Sigma_{\mathrm{u}} \rightarrow \mathrm{X}^{2} \Sigma_{\mathrm{g}}((1,1)$ & 3884.3 & 1700 \\
$\mathrm{NH}$ & $\mathrm{C}^{1} \Pi \rightarrow \mathrm{B}^{1} \Sigma(0,0)$ & $4504.0^{*}$ & 2500 \\
$\mathrm{NH}$ & $\mathrm{A}^{3} \Pi \rightarrow \mathrm{X}^{3} \Sigma(0,0)$ & 3360 & - \\
$\mathrm{NH}$ & $\mathrm{A}^{3} \Pi \rightarrow \mathrm{X}^{3} \Sigma(1,1)$ & 3371 & - \\
${ }^{*} Q$ head, $\mathrm{R}$ head at 4525 $\AA$ and P head at $4538 \AA$ \\
\hline
\end{tabular}

Because the relaxation times for rotational equilibration are of the same order as that for adjustment of the translational motions of molecules, ${ }^{18.19}$ (i.e. only a few collision are needed), in the absence of local thermodynamic equilibrium, the rotational temperature may provide a good estimate of the kinetic temperature of a molecule. However, if collisional rates are insufficient to provide equilibrating rotational energy transfer, the molecules are rotationally frozen and the rotational temperature reflects the kinetic temperature at the point the molecules were frozen. No attempt was made to estimate the collision frequencies for the molecular constituents due to the difficulty in finding accurate estimates of the collisional cross sections, however, the relatively low rotational temperature values determined for $\mathrm{N}_{2}$ at the nozzle exit plane compared to estimates of the rotational temperature in the constrictor suggest the nitrogen was not rotationally frozen. Therefore, the temperature of approximately $750 \mathrm{~K}$ reflects the degree to which the nitrogen was cooled in the gasdynamic expansion.

The rotational temperature for the molecular nitrogen ions was determined to be approximately $1000 \mathrm{~K}$ hotter than the molecular nitrogen. Because the electron - ion collisional cross sections are much larger than the electron - neutral collisional cross sections, heating via electronic collisions occurred more frequently for $\mathrm{N}_{2}{ }^{+}$. Therefore, the rotational energy distribution for $\mathrm{N}_{2}{ }^{+}$reflected a higher temperature than for $\mathrm{N}_{2}$. The rotational temperature for the NH molecules was substantially higher than the values for $\mathrm{N}_{2}$ and $\mathrm{N}_{2}{ }^{+}$. As previously indicated, the $\mathrm{NH}$ molecules resulted from recombination of dissociated hydrogen and nitrogen within the nozzle This recombination could occur as the result of two body collisions or three body collisions involving 
electrons, heavy particles, or the nozzle walls. The rates of radiative recombination, (i.e. two body recombination), and three body recombination were estimated based on recombination coefficients presented in Mitchner and Kruger. ${ }^{20}$ These calculations indicate the dominant recombination mechanism was three body recombination. In this case, some or all of the recombination energy was carried off as increased kinetic energy of the collisional partner or as heating of the nozzle walls. The remainder of the recombination energy remained with the recombined molecule. With sufficient vibrational-rotational energy transfer, a portion of the recombination energy increased the molecule's. rotational temperature. Therefore, the recombined NH molecule was rotationally hotter than the other molecular constituents.

Electrical Configuration. The electrical configuration had a measurable effect on the overall intensities of the observed $\mathrm{H}_{\alpha}, \mathrm{H}_{\beta}$, and $\mathrm{NH}$ transitions. For example, the inverted $\mathrm{H}_{\alpha}$ radial intensity profiles from the nozzle exit plane for each configuration are shown in Figure 9. The intensities at configuration 2 were more than three times brighter than those measured for configuration 1 and more than 20 times brighter than those for configuration 3 . Similar results were obtained at other axial positions for the $H_{\alpha}, H_{\beta}$, and $\mathrm{NH} \mathrm{A}^{3} \Pi \rightarrow \mathrm{X}^{3} \Sigma$ transitions. The lower intensities for configuration 1 and configuration 3 are the result of a lower population of the excited states. This suggests that significant population of excited states is occurring only within the current attachment regions of the anode.

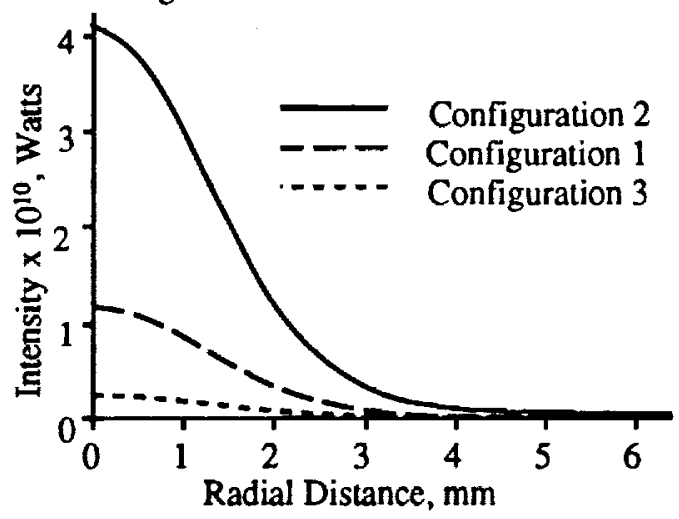

Figure 9: Inverted $H_{\alpha}$ Intensities at the Exit Plane for Each Electrical Configuration
The changes in the measured intensity for the different electrical configurations were attributed to changes in the rate of electron collisional excitation within the nozzle, which determines the population of excited states. The rate of collisional excitation depends on the number density of the species being excited, the electron number density, the electron energy distribution, and the appropriate collisional cross sections. There were significant changes in the distribution of the current carrying electrons in the nozzle with configuration. A summary of the current distribution and overall power for each configuration is shown in Table IV. Based on spectroscopic measurements inside the anode of a similar laboratory-model arcjet the electron number density inside the nozzle decreases from approximately $10^{15}$ $\mathrm{cm}^{-3}$ near the constrictor to approximately $10^{14} \mathrm{~cm}^{-3}$ near the nozzle exit.21 However, the number of $1 \mathrm{eV}$ electrons needed to carry 10 Amperes of current is approximately $10^{11} \mathrm{~cm}^{-3}$. Thus, changes in the distribution of current carrying electrons likely had a small effect on the overall electron number densities inside the nozzle.

Since the electron number density does not change significantly with electrical configuration, it is reasonable to assume that changes in the electron energy distribution function were responsible for the observed changes in the excitation spectrum. For example, by changing from configuration 2 to configuration 3 , the electron acceleration due to the electric field is forced to occur in a higher pressure region of the nozzle. Thus, more collisional energy exchange between the electrons and heavy particles occurred in configuration 3 limiting the number of energetic electrons available for collisional excitation. In configuration 2 the electron acceleration due to the electric field continues downstream to segment 5 . In this region there was less collisional energy exchange with heavy particles due to lower total number densities. Consequently, the electrons were accelerated to higher velocities than in configurations 1 and 2 and, more energetic electrons were available for collisional excitation

In configuration 3 , the electrons were no longer accelerated due to the electric field in the region of the anode corresponding to segment 5 . Therefore, in this region the electron collisional excitation depleted the high energy electrons. Electron self equilibration

Table IV: Voltage/Current Characteristics

\begin{tabular}{|c|c|c|c|c|c|c|c|c|c|}
\hline & Segment I & \multicolumn{2}{|c|}{ Segment 2} & \multicolumn{2}{|c|}{ Segment 3} & \multicolumn{2}{|c|}{ Segment 4} & \multicolumn{2}{|c|}{ Segment 5} \\
\hline voltage & current & voltage & current & voltage & current & voltage & current & voliage & current \\
\hline Configuration 154 & 0.0 & 135 & 2.0 & 135 & 1.9 & 135 & 1.9 & 135 & 4.3 \\
\hline Configuration 255 & 0.0 & 100 & 0.0 & 110 & 0.0 & 125 & 0.0 & 140 & 10.0 \\
\hline Configuration 3138 & 0.4 & 138 & 4.4 & 138 & 4.4 & 138 & 0.8 & 122 & 0.0 \\
\hline
\end{tabular}


rapidly seeks to reestablish a Maxwellian energy distribution, however, at a slightly reduced temperature. A lower electron energy distribution further reduces the rate of electron collisional excitation. It seems that this electron cooling mechanism occurred with such rapidity that the rate of collisional excitation quickly approached the rate of radiative decay within this part of the nozzle. This resulted in the more pronounced deviation from equilibrium for the excited states of atomic hydrogen indicated by negative temperature values calculated using the line intensity ratio for configuration 3 .

Erozen Flow Losses. In the arcjet, electrons are accelerated by the electric field and impart energy to the propellant via collisional processes. Some of this energy is invested in excitation, ionization and molecular dissociation. The energy that remains in these internal modes at the nozzle exit is called the frozen flow loss. Based on the overall device efficiency and reasonable estimates of other loss mechanisms it is likely that frozen flow losses account for 30 to 40 percent of the total energy input to the device. 22

The variation in the measured intensities demonstrated that the location of the current attachment on the anode significantly effects excited state number densities in the plume. Results reported in a companion paper indicate that the location of the current attachment on the anode had a negligible effect on the measured thrust of the device. ${ }^{10}$ These results imply that the frozen flow losses due to electronic excitation were negligible. Because 12.1.and $12.7 \mathrm{eV}$ are required to excile the third and fourth energy levels of hydrogen, the upper excited states of $H_{\alpha}$ and $H_{\beta}$, this result has significant implications about other frozen flow losses in kilowatt-class arcjets.

Consider the ionization and dissociation energies shown in Table V. Recent measurements indicate that the electron temperature in the anode is below 2 $\mathrm{eV}^{21}$ If the electron energy distribution for this temperature is Maxwellian, in the absence of stepwise excitation, the electrons in the high energy tail were responsible for ionization and the excitation of upper excited states with energies approaching the ionization potential, such as $\mathrm{H}_{\alpha}$ and $\mathrm{H}_{\beta}$. The fraction of electrons in this high energy tail is small, (eg. the fraction of electrons having energies above $13.6 \mathrm{eV}$ is 0.005 for a $2 \mathrm{eV}$ electron temperature). Given that significant changes in the frozen flow losses due to excitation of hydrogen were insignificant from a performance standpoint, it is unlikely that other processes requiring these energetic electrons were dominant. Therefore, ionization, although integral to the operation of the arcjet, was responsible for only a small part of the frozen flow losses, because of the high energy thresholds relative to the electron temperature. Ionization losses of approximately 10 Watts, or less than 1 percent of the total energy input, were estimated. This estimate was based on the assumption of quasi-neutrality, (ie. the ion number density approximately equal to the electron number density), an ion exit velocity of $5000 \mathrm{~m} / \mathrm{s}$, based on the measured specific impulse, and an average ionization energy of $14 \mathrm{eV}$. For ionization losses on the order of 10 Watts, less than one percent of the flow was ionized. This agrees with the ionization fraction reported by Zana. ${ }^{5}$

Table V. Ionization and Dissociation Energies

\begin{tabular}{ccc}
\hline Species & Ionization *,eV & Dissociation, $\mathrm{eV}$ \\
\hline $\mathrm{H}$ & 13.60 & - \\
$\mathrm{H}_{2}$ & 15.48 & 4.53 \\
$\mathrm{~N}$ & 14.53 & $-\overline{7}$ \\
$\mathrm{~N}_{2}$ & 15.58 & 9.85 \\
$\mathrm{NH}$ & 13.10 & 3.26 \\
& * first ionization state \\
\hline
\end{tabular}

Therefore, because frozen flow losses due to electronic excitation and ionization were small, the dominant frozen flow loss mechanism was dissociation. This is because the energy thresholds for dissociation are much closer to the electron temperature. As a result, the number of electrons capable of collisional dissociation is relatively large, (e.g. the fraction of electrons with energies above the $4.5 \mathrm{eV}$ threshold for dissociation of hydrogen is 0.2 for a $2 \mathrm{eV}$ electron temperature). A smaller number of electrons have energies above the $9.9 \mathrm{eV}$ dissociation energy of nitrogen. Minimal amounts of atomic nitrogen were detected at the exit plane. The presence of excited $\mathrm{NH}$ in the emission spectra indicated that significant amounts of atomic nitrogen had recombined in the nozzle. However, due to the small dissociation energy of the NH molecule, this recombination recovered only some of the encrgy lost due to dissociation. A total of over $14 \mathrm{eV}$ is required to dissociate both a hydrogen and a nitrogen molecule; however, only $6.5 \mathrm{eV}$ are recovered by two NH recombinations.

\section{Conclusions}

Light intensities resulting from the de-excitation of electronically excited species in the near field of the plume were measured. Excitation temperature, rotational temperature, and electron number density were evaluated based on these intensity measurements. The effect the anode current distribution had on the characteristics of the plume was investigated.

The complete emission spectrum, measured from $3200 \AA$ to $7200 \AA$ at the nozzle exit plane detected the electronically excited species $\mathrm{N}_{2}, \mathrm{~N}_{2}{ }^{+}, \mathrm{NH}$, and $\mathrm{H}$, indicating excitation, dissociation, ionization and 
recombination in the nozzle. Radial intensity profiles, spaced $0.05 \mathrm{~mm}$ apart, were measured for the $\mathrm{H}_{\alpha}, \mathrm{H}_{\beta}$, and $\mathrm{NH} \mathrm{A}^{3} \Pi \rightarrow \mathrm{X}^{3} \Sigma(0,0)$ transitions at four different axial locations. These line of sight intensities measurements were deconvoluted to give the radial intensity distribution using an inverse Abel transformation. Axial intensity profiles taken on centerline indicated an exponential decay in excited state population for $\mathrm{H}_{\alpha}, \mathrm{H}_{\beta}$, and $\mathrm{NH}$. The rate of axial decay indicated lower velocities for $\mathrm{NH}$ than $\mathrm{H}$ in the plume and population of the third excited energy state of hydrogen from the decay of higher energy levels. The suitability of a line intensity ratio method, which requires a Boltzmann energy distribution between excited states for the determination of an excitation temperature, was evaluated. In some instances, this method resulted in negative temperatures. This indicated a nonequilibrium energy distribution between excited energy states of hydrogen in the plume. This was analytically verified. Rotational temperatures evaluated based on the emission spectra for several molecular transitions ranged from $750 \mathrm{~K}$ for $\mathrm{N}_{2}$ to $2500 \mathrm{~K}$ for NH. Based on these results, the arcjet plume was found to be a highly non-equilibrium plasma.

Anode electrical configuration was found to have a large effect on the spectral intensities measured in the plume. These data indicate that significant population of excited states occurs mainly in the current attachment regions in the nozzle. Therefore, by forcing the arc to attach farther upstream, frozen flow losses losses due to electronic excitation were significantly reduced. The reduction in the frozen flow losses due to excitation was determined to be negligible from a performance standpoint based on thrust measurements reported in a companion paper. ${ }^{10}$ As a result, the dominant frozen flow loss mechanism in kilowatt class arcjets run on hydrogen/nitrogen mixtures was dissociation.

\section{References}

1. Knowles, S.K., Smith, W.W., Curran, F.M., and Haag, T.W., "Performance Characterization of a Low Power Hydrazine Arcjet," AIAA 871057, May 1987.

2. Curran, F.M. and Haag, T.W., "An Extended Life and Performance Test of a Low Power Arcjet," AIAA 88-3106, July 1988, (NASA TM100942).

3. Yano, S. and Knowles, S.K., "Simulated Flight Qualification Test of an Engineering Model Arcjet System," presented at the 1989 JANNAF meeting, Cleveland, OH, May 1989.

4. Carney, L.M. and Sankovic, J.M., "The Effects of Arcjet Thruster Operating Conditions and Constrictor Geometry on the Plasma Plume,"
AIAA 89-2723, July 1989, (NASA TM102284).

5. Zana, L.M., "Langmuir Probe Surveys of an Arcjet Exhaust," AIAA 87-1950, July 1987, (NASA TM-89924).

6. Ling, H., et al., "Reflector Performance Degradation due to an Arcjet Plume," presented at the 1989 Antenna Applications Symposium, Monticello, II, Sept. 1989.

7. Curran, F.M., Sovie, A.J., and Haag, T.W., "Arcjet Nozzle Design Impacts," presented at the 1989 JANNAF meeting, Cleveland, OH, May 1989.

8. Curran, F.M. and Manzella, D.H., "The Effect of Electrode Configuration on Arcjet Performance," AIAA 89.2722, July 1989, (NASA TM102346).

9. Hardy, T.L., and Curran, F.M., "Low Power dc Arcjet Operation with Hydrogen/Nitrogen/Ammonia Mixtures," AIAA 87-1948, June 1987, (NASA TM-89876).

10. Curran, F.M., Manzella, D.H., and Pencil E.J., "Performance Characterization of a Segmented Anode Arcjet Thruster," AIAA 90-2582, July 1990.

11. Griem, H.R., Plasma Spectroscopy, McGraw Hill, New York, 1964.

12. Wiese, W.L., "Line Broadening," in Plasma Diagnostic Techniques, Huddlestone, R.H. and Leonard, S.C., eds., Academic Press, New York, 1965.

13. Herzberg, G., Molecular Spectra and Molecular Structure I. Spectra of Diatomic Molecules, 2nd ed., D. Van Nostrand Company, Inc., Princeton, New Jersey, 1950.

14. Larrabee, R.D., "Spectral Emissivity of Tungsten," J. Opt. Soc. Am., Vol 49, No.6, p. 619, June 1959.

15. Wiese, W.L., Smith, M.W., and Glennon, B.M., "Atomic Transition Probabilities: Hydrogen through Neon," NSRDS-NBS4 Vol I, May 1966.

16. Pearse, R.W.B. and Gaydon, A.G., The Identification of Molecular Spectra, John Wiley \& Sons Inc. New York, 1950.

17. Drawin, H., "Collision and Transport CrossSections," EUR-CEA-FC-383, 1966.

18. Vincentti, W.G. and Kruger, C.H., Introduction to Physical Gas Dynamics, Robert E. Krieger Publishing Co., New York, 1967.

19. Park, C., Nonequilibrium Hypersonic Aerothermodynamics, John Wiley and Sons, New York, 1990.

20. Mitchner, M. and Kruger, C.H., Partially lonized Gases, John Wiley and Sons, New York, 1973.

21. Zube, D.M., "Emission Spectroscopy Experiments in a Low Power Arcjet Nozzle," Diplom Ingenieur Thesis, University of Stuttgart, Stuttgart, West Germany, 1990. 
22. Jack,J.R., "Theoretical Performance of Propellants Suitable for Electrothermal Jet Engines," NASA TN D-682, March 1961.

23. Sudharsanan, S.I., "The Abel Inversion of Noisy Data Using Discrete Integral Transforms," Master's Thesis, University of Tennessee, Knoxville, Tennessee, 1986.

24. Oppenheim, A.V. and Schafer, R.W., Digital Signal Processing, Prentice-Hall, Englewood Cliffs, New Jersey, 1975.

\section{Appendix}

Abel inversion The spectral intensities measured in this investigation were line of sight values, measured by looking through the plume from the side, perpendicular to the arcjet center line. For line of sight measurements, the intensity values correspond to integrated values of intensity as a function of position. If the plume is axisymmetric, the emission coefficient at any point, $\varepsilon$, is only a function of the radial coordinate, $r$. This situation is depicted schematically in Figure 10.

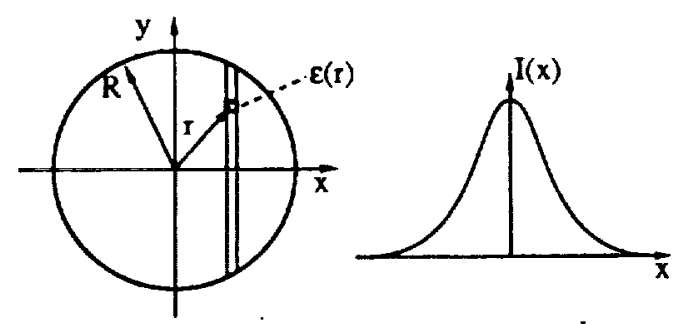

Figure 10: Projection of an Axisymmetric Function

If there is no significant reabsorption of the emitted radiant energy in the plume, it is optically thin. For an axisymmetric, optically-thin plume the observed intensity at a location $x$ is given by:

$$
I(x)=\int_{-\infty}^{\infty} E(r) d y=\int_{-\infty}^{-} \varepsilon\left(\sqrt{x^{2}+y^{2}}\right) d y
$$

There are several methods to determine the emission coefficient, $\varepsilon(r)$, from Equation (A1). The methods for reconstructing this radially dependent emission coefficient from the projected intensity values are known as inverse Abel transformations. Several techniques have been used to perform inverse Abel inversions. The method presented here is based on that originally presented by Sudharsanan. ${ }^{23}$ The algorithms utilized and the peak finding routine were independently derived and are presented here. The Fourier transform of Equation (A1) results in the following expression:

$$
G(f)=\int_{-\infty}^{\infty} I(x) e^{-j 2 \pi x} d x
$$

By changing to polar coordinates and substituting for the zeroth order Bessel function, it can be shown that equation (A2) is equal to the zeroth order Hankel transform of $\varepsilon(r) .{ }^{23}$ Therefore, the emission coefficient can be obtained from the inverse Hankel transform.

$$
\varepsilon(r)=\int_{0}^{\infty} G(f) f J_{0}(2 \pi f r) d r
$$

In actual practice the intensities are only available at discrete positions and the center line of the data is not known. This is accommodated using a discrete Fourier representation of the projected intensity values. The Fourier transform can be evaluated using the following direct Fourier transform (DFT) algorithm:

$$
\widehat{G}_{1}=\sum_{i=0}^{N 1} \mathrm{I}(i) \exp \left(\frac{-2 \pi i l}{N}\right)
$$

In order to evaluate the emission coefficient, the inverse Hankel transform is performed. Since the intensity profile is axisymmetric, the inverse Hankel transform need only be calculated for half the intensity profile. However, to do this the transformed intensity data must be shifted such that the axis of symmetry is at the origin. For noise-free data correctly centered on the interval $\mathrm{i}=0$ to $\mathrm{N}-1$, the imaginary part of the DFT is zero. The magnitude of the shift needed to properly center the data can be determined by minimizing the imaginary component of the DFT of the actual data. The values calculated using the algorithm in equation (A4) are then shifted the appropriate amount using the DFT shift theorem ${ }^{24}$ to place the center line at $i=0$. The emission coefficient is then evaluated using the following discrete representation:

$$
\hat{\varepsilon}(r)=\frac{2 p}{N^{2}(\Delta x)} \sum_{i=0}^{N / 2-1} \widehat{G}(1) 1 J_{0}\left(\frac{2 \pi r l}{N}\right)
$$

Figure 11 shows the inverted results for two analytic test functions. Figure 11(a) shows inverted values for an ellipse. The exact solution is a step function. There is ringing in the calculated solution at the singularity. However, this type of profile would not likely be encountered and is included only as a worst case. The solution shown in Figure 11(b) is for a Gaussian input function. The exact solution and the calculated values are coincident. This is closer to the cases expected in emission spectroscopy experiments. 


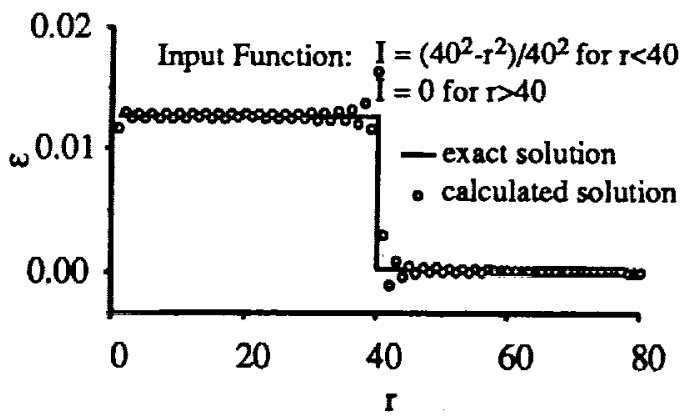

(a) Step Function

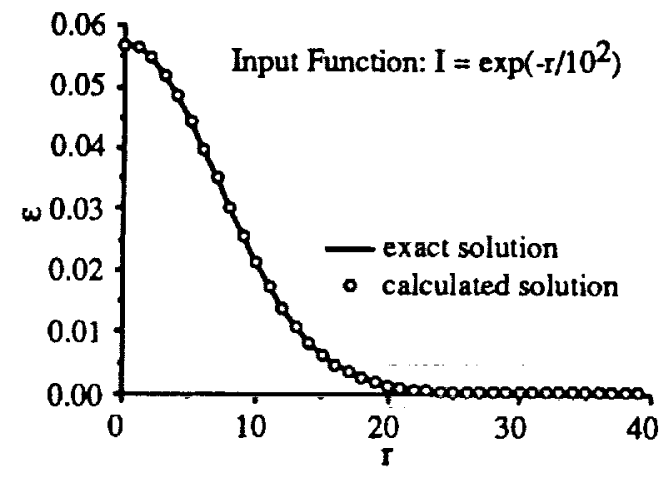

Gaussian Function

Figure 11: Verification of Inverse Abel Transformation with Analytic Functions 


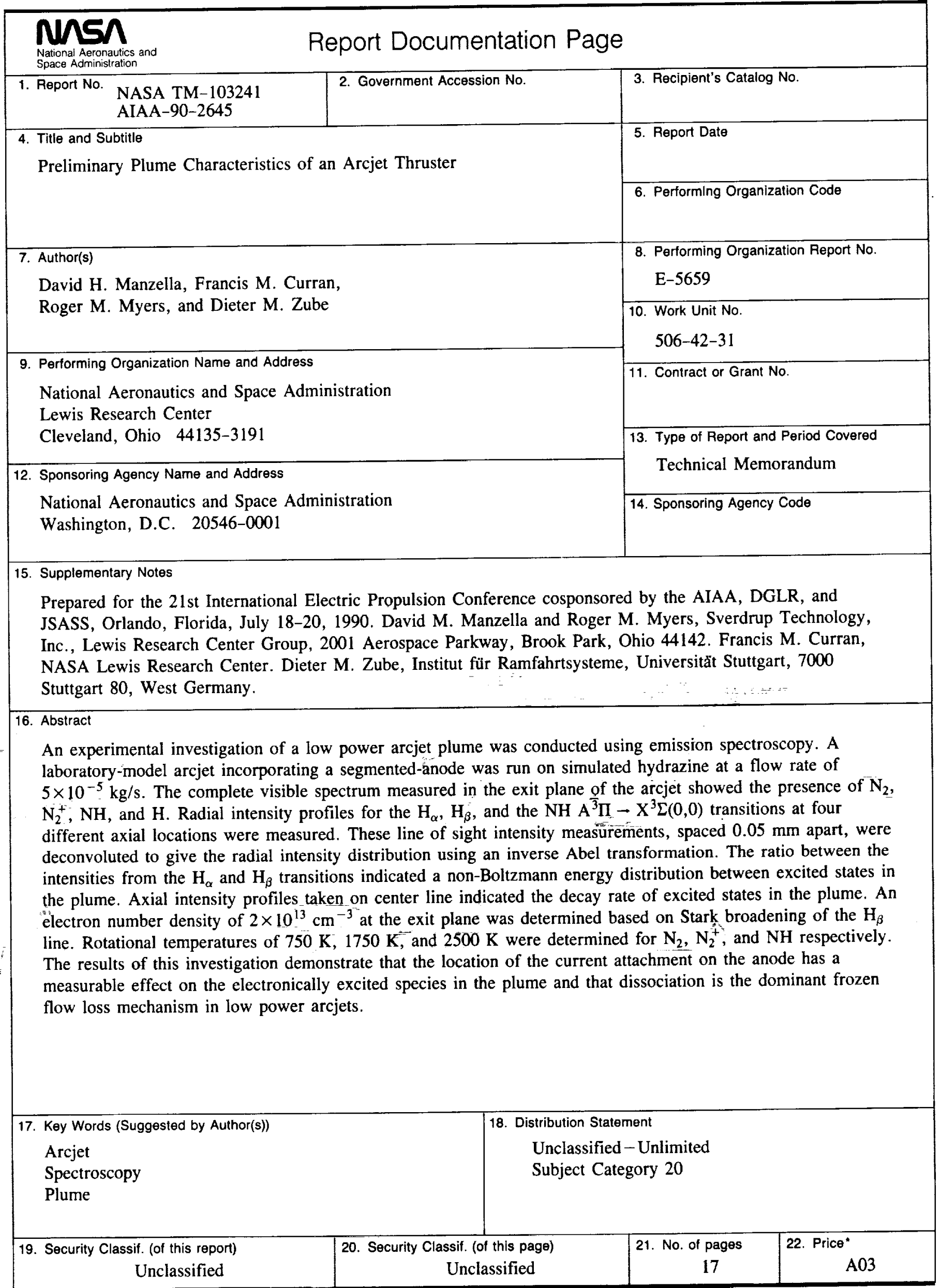

NASA FORM 1626 ○CT $86 \quad$ *For sale by the National Technical Information Service, Springfield, Virginia 22161 
